\title{
Evaluation of Medication Use Patterns among Geriatric Patients using World Health Organization Prescribing Indicators
}

\author{
Irma M. Puspitasari, Ani Hanifah, Rano K. Sinuraya \\ Department of Pharmacology and Clinical Pharmacy, Faculty of Pharmacy, \\ Universitas Padjadjaran, Bandung, West Java, Indonesia
}

\begin{abstract}
Geriatrics patients are paticularly suceptible to medication error due to complex clinical problems and multiple treatment. World Health Organization (WHO) published a set of prescribing indicators to promote rationale use of drug. This study aimed to evaluate medicine use pattern in geriatric patients at a primary care facility in Bandung, Indonesia, using WHO indicators. This research was conducted using cross sectional design with retrospective data collection. Medical presciption from geriatric patients aged 60-74 years old visiting primary care facility during 2013-2015 were selected. A total of 103.292 prescriptions were obtained in this study. The average number of drugs per encounter was 2.1 (optimal range=1.6-1.8). Vast majority $(99.41 \%)$ of the drugs prescribed were generic drugs (optimal value $=100 \%$ ). The encounters with an antibiotics prescribed were $7.4 \%$ (optimal range $=20.0-26.8 \%$ ). Very few parenteral drugs were prescribed in this study setting $(0.04 \%)$ (optimal range $=13.4-24.1 \%$ ). The drugs prescribed from essential drug list were $72.83 \%$ (optimal value $=100 \%$ ). The most commonly prescribed drugs were paracetamol $500 \mathrm{mg}$, chlorpheniramine maleat $4 \mathrm{mg}$, amlodipine $5 \mathrm{mg}$, vitamin B complex, and glyceryl guaiacolat. The most frequently prescribed antibiotics were amoxicillin, cloramphenicol, cyprofloxacin, clindamycin, and oxytetracyclin. In conclusion, the medication use pattern in this study was below the WHO requirement. This finding called for a strategy to promote rational prescribing of medicines.
\end{abstract}

Keywords: geriatrics, WHO prescribing indicators, drug use patterns.

\section{Introduction}

Appropriate use of medicine is essential to optimize outcome of the therapy and increase quality of life of the patients. Several aspects that need to be considered in providing good quality patient care include accurate diagnosis, an effective prescribing system, and affordable costs. Nevertheless, previous studies showed

that $50 \%$ of prescribing practice worldwide is still irrational. ${ }^{1-3}$

Irrational prescribing of drugs can result in unsafe and ineffective treatment, nonadherence towards medication, increased morbidity and mortality, prolongation of

Corresponding author: Irma M. Puspitasari. Department of Pharmacology and Clinical Pharmacy, Faculty of Pharmacy, Universitas Padjadjaran. Email: irma.melyani@unpad.ac.id

Received: 13 June 2019. Revised: 2 August 2019 Published: 30 August 2019. 
disease, and higher health care cost. The crucial step to limit irrational use of medicine is to identify the type, quantity, and severity of irrational drug use.,

Geriatric patients are particularly vulnerable to the risk of medication-related problem due to age-related physiological changes that might affect pharmacokinetics and pharmacodynamics of drugs. Besides, multiple medical condition might lead to complex therapeutic regimen for elderly patients. $^{6}$

The World Health Organization (WHO) published a prescribing indicator to promote rational prescribing of medicine. This indicator can be used to detect problems in the prescribing practice, such as polypharmacy, excessive use of antibiotics and injections, prescribing of branded drugs, and disobedience to clinica practice guideline. ${ }^{4-5}$. In addition, this indicator also can be used as monitoring tool to detect problems in the performance of health care providers. ${ }^{7}$

Currently, there is limited information on the quality of medication prescribing for geriatric patients in Indonesia. Thus, this study was conducted to investigate medicine use pattern amongg eriatric patients visiting a primary care facility in Bandung, Indonesia during 2013-2015.

\section{Methods}

The conduct of this study was approved by the Health Research Ethics Committee of the Faculty of Medicine, Padjadjaran University (No. 424 / UN6.C.10 / PN / 2017) and Department of Health, Bandung City (No. 070 / 3398-DHO).

This research was conducted at a primary care facility in Bandung, Indonesia. This study used cross sectional design with retrospective data collection. Medical presciption from geriatric patients aged 60-74 years old visiting primary care facility during 2013-2015 were selected. In this study, geriatric patients were defined as patients aged 60 years to 74 years according to the Indonesian Ministry of Health 2009 The National Essential Medicines List (DOEN) used is the DOEN issued in 2011 by the Ministry of Health.

The study population was geriatric patients in primary care facilities in Bandung. The target population was geriatric patients at a primary care facility in Bandung during 2013-2015. The criteria for research subjects include:

1. Inclusion criteria

Electronic prescriptions for patients aged 60-74 years old during 2013-2015.

2. Exclusion criteria

a. Patients aged 60-74 years who did not receive medication or only referred to the hospital.

b. Incomplete patient data.

Table 1. Diagnosis of Geriatric Patients in Primary Care Facilitiy during 2013-2015

\begin{tabular}{lcccc}
\hline \multicolumn{1}{c}{ Diagnosis } & $\mathbf{2 0 1 3}$ & $\mathbf{2 0 1 4}$ & $\mathbf{2 0 1 5}$ & Total \\
\hline Primary hypertension & 1089 & 1039 & 1526 & 3654 \\
Acute respiratory tract infection & 351 & 372 & 479 & 1202 \\
Myalgia & 494 & 357 & 340 & 1191 \\
Diabetes mellitus & 221 & 273 & 298 & 792 \\
Pulp and periapical disease & 174 & 141 & 242 & 557 \\
\hline
\end{tabular}


Table 2. Assessment of Drugs Prescribing based on WHO Indicator

\begin{tabular}{|c|c|c|c|c|c|c|}
\hline \multirow{2}{*}{ No } & \multirow{2}{*}{ Indicator } & \multicolumn{3}{|c|}{ Result } & \multirow{2}{*}{$\begin{array}{l}\text { Standar } \\
\text { WHO }\end{array}$} & \multirow{2}{*}{ P-value } \\
\hline & & 2013 & 2014 & 2015 & & \\
\hline 1 & Average number of drugs per encounter & 2.4 & 1.9 & 2.1 & $1.6-1.8$ & 0.000 \\
\hline 2 & $\begin{array}{l}\text { Percentage medicines prescribed by } \\
\text { generic names }\end{array}$ & $99.60 \%$ & $99.17 \%$ & $99.46 \%$ & $100 \%$ & 0.000 \\
\hline 3 & $\begin{array}{l}\text { Percentage encounters with antibiotic } \\
\text { prescribed }\end{array}$ & $7.2 \%$ & $7.3 \%$ & $7.7 \%$ & $20-25.4 \%$ & 0.635 \\
\hline 4 & $\begin{array}{l}\text { Percentage encounters with injection } \\
\text { prescribed }\end{array}$ & $0.02 \%$ & $0.02 \%$ & $0.06 \%$ & $10-17 \%$ & 0.547 \\
\hline 5 & $\begin{array}{l}\text { Percentage medicines prescribed from } \\
\text { Essential Drug List }\end{array}$ & $68.45 \%$ & $73.91 \%$ & $76.15 \%$ & $100 \%$ & 0.000 \\
\hline
\end{tabular}

From each prescriptions, the following information were obtained; patient category, date, age, diagnosis, amount of medication, generic drugs, antibiotic drugs, injection drugs, essential drugs (if any). The data was then statistically analyzed using one way ANOVA and Bonferroni post hoc-test with EZR Program version 1.35P. $\mathrm{P}<0.05$ defined statistical significance.

\section{Results and Discussion}

A total of 103.292 prescriptions were obtained in this study. The average number of drugs per encounter was 2.1 (optimal range=1.6-1.8). Vast majority (99.41\%) of the drugs prescribed were generic drugs (optimal value $=100 \%$ ). The encounters with an antibiotics prescribed were $7.4 \%$ (optimal range $=20.0-26.8 \%$ ). Very few parenteral drugs were prescribed in this study setting $(0.04 \%)$ (optimal range $=13.4-24.1 \%)$. The drugs prescribed from essential drug list were $72.83 \%$ (optimal value $=100 \%$ ). The most commonly prescribed drugs were paracetamol $500 \mathrm{mg}$, chlorpheniramine maleat $4 \mathrm{mg}$, amoxicillin $500 \mathrm{mg}$, and thiamin $\mathrm{HCl}$. The most frequently prescibed antibiotics were amoxicillin, cloramphenicol, cyprofloxacin, clindamycin, and oxytetracyclin.

\section{Average number of drugs per encounter}

The purpose of this indicator is to measure the level of polypharmacy. According to the WHO, the recommended average number of drugs per encounter was 1.6-1.8. In this study, the average number of drugs per encounter was 2.1. The occurrence of polypharmacy could increase the risk of unwanted side effects, drug interactions, and non-adherence to medication. ${ }^{7,8}$ Nevertheless, in geriatric patients, multiple medication might be considered necessary to treat multiple medical condition.

Table 3. The Most Frequently Prescribed Drugs

\begin{tabular}{lcccc}
\hline \multicolumn{1}{c}{ Name of Drugs } & $\mathbf{2 0 1 3}$ & $\mathbf{2 0 1 4}$ & $\mathbf{2 0 1 5}$ & Total \\
\hline Paracetamol 500 mg & 1654 & 1720 & 1804 & 5178 \\
Chlorpheniramine maleat 5 mg & 708 & 638 & 694 & 2040 \\
Amlodipine 5 mg & 178 & 388 & 1324 & 1890 \\
Vitamin B complex & 451 & 285 & 791 & 1527 \\
Glyceryl guaiacolat $100 \mathrm{mg}$ & 659 & 117 & 359 & 1135 \\
\hline
\end{tabular}


Table 4. The Most Commonly Prescribed Antibiotics

\begin{tabular}{lcccc}
\hline \multicolumn{1}{c}{ Antibiotic } & $\mathbf{2 0 1 3}$ & $\mathbf{2 0 1 4}$ & $\mathbf{2 0 1 5}$ & Total \\
\hline Amoxicillin & 243 & 263 & 275 & 781 \\
Cloramphenicol & 13 & 17 & 54 & 84 \\
Cyprofloxacin & 24 & 5 & 16 & 45 \\
Clindamycin & 14 & 13 & 0 & 27 \\
Oxytetracyclin & 17 & 5 & 0 & 22 \\
\hline
\end{tabular}

Percentage medicines prescribed by generic names

The purpose of this indicator is to measure the tendency to prescribe drugs with generic name. Generic prescribing is cost effective and associated with less potential of error., ${ }^{9,10}$ The WHO recommended $100 \%$ of medicines in health care facilities are prescribed by generic name. The result of this study (99.41\%) was relatively in accordance with WHO standard.

Percentage encounters with antibiotic prescribed Excessive use of antibiotics was associated with incresed risk of antibiotic resistance. The purpose of this indicator is to measure the antibiotic use in health care facility. ${ }^{7}$ In this study, the percentage of encounters with antibiotics prescribed was $7.2 \%$ in 2013 , $7.3 \%$ in 2014 and $7.7 \%$ in 2015 . It was below the WHO standard (20-25.4\%). This might be due to the competency of the prescribers and the presence of national guideline on the use of antibiotics. It was relatively low compared to Cameroon (36.7\%) and Pakistan $(22.1 \%){ }^{11,12}$
The most widely used antibiotic in this study setting was Amoxicilin $500 \mathrm{mg}$, with a total of 781 set of drugs prescribed from 2013-2015. Amoxicillin is a broad spectrum antibiotics that is used to treat several infectious disease in respiratory tract, gastrointestinal, urinary tract, and skin ${ }^{13}$.

Percentage encounters with injection prescribed In this study, the prescribing of injection was relatively low. Excess and unsafe use of injections can result in potential harm to the patients and wasteful cost, compared to oral preparations. In this indicator, immunication was not counted as an injection. ${ }^{14}$

Percentage medicines prescribed from Essential Drug List

The drugs prescribed from essential drug list were $72.83 \%$ (optimal value $=100 \%$ ). The purpose of this indicator is to measure the adherence of prescribing practice to national guideline. Essential drug list are drugs that satisfy priority health care need for population. contains the most effective and safe drugs that meet clinical needs of the population. ${ }^{15}$

Table 5. Injection Drugs Prescribed

\begin{tabular}{ccccc}
\hline Injection Drugs & $\mathbf{2 0 1 3}$ & $\mathbf{2 0 1 4}$ & $\mathbf{2 0 1 5}$ & Total \\
\hline Cyanocobalamine injection & 1 & 0 & 0 & 1 \\
\hline Antalgin inj $250 \mathrm{mg} / \mathrm{ml}-2 \mathrm{ml}$ & 0 & 1 & 0 & 1 \\
\hline
\end{tabular}




\section{Conclusion}

The medication use pattern in this study was below the WHO requirement. This finding called for a strategy to promote rational prescribing of medicines.

\section{Acknowledgement}

None declared.

\section{Funding}

None

\section{Conflict of Interest}

None declared.

\section{References}

1. Ofori-Asenso R, Agyeman AA. Irrational use of medicines:A summary of key concepts. Pharmacy (Basel). 2016;4(4):35.

2. Kshirsagar NA. Rational use of medicines: Cost consideration \& way forward. Indian Journal of Medical Research. 2016;144(4):502-505.

3. Lionis C, Petelos E, Shea S. Irrational prescribing of over-the-counter (OTC) medicines in general practice: testing the feasibility of an educational intervention among physicians in five European countries. BMC Family Practice. 2014;15:34.

4. Barkus A, Lisauskienè I. Inappropriate habits of antibiotic use among medical specialists and students in Vilnius. Acta Medica Lituania. 2016;23(2):135-141.

5. Sköldunger A, Fastbom J, Wimo A. Impact of inappropriate drug use on hospitalizations, mortality, and costs in older persons. Drugs Aging. 2015;32(8):671-678

6. Pérez-Jover V, Mira JJ, CarratalaMunuera C. Inappropriate use of medication by elderly, polymedicated, or multipathological patients with chronic diseases. International Journal of Environmental Research and Public Health. 2018;15(2):310.

7. Karimi A, Haerizadeh M, Soleymani F. Evaluation of medicine prescription pattern using World Health Organization prescribing indicators in Iran: A crosssectional study. Journal of Research in Pharmacy Practice. 2014;3(2):39-45.

8. Abdulah R, Insani WN, Destiani DP, Rohmaniasari N. Polypharmacy leads to increased prevalence of potentially inappropriatemedication in the Indonesian geriatric population visiting primary care facilities. Therapeutic Clinical Risk Management. 2018;14:1591-1597.

9. Andrade C, Rao TSS. Prescription writing: Generic or brand?. Indian Journal of Psychiatry. 2017;59(2):133137.

10. Roy V, Rana P. Prescribing generics: All in a name. Indian Journal of Medical Research. 2018;147(5):442-444.

11. Chem ED, Anong DN, Akoachere JKT. Prescribing patterns and associated factors of antibiotic prescription in primary health care facilities of Kumbo East and Kumbo West health districts, North West Cameroon. PLoS One. 2018;13(3):e0193353.

12. Atif M, Azeem M, Sarwar MR. WHO/ INRUD prescribing indicators and prescribing trends of antibiotics in the accident and emergency department of Bahawal Victoria Hospital, Pakistan. Springerplus. 2016;5(1):1928.

13. Stolbrink M, Bonnett LJ, Blakey JD. Amoxicillin is associated with a lower risk of further antibiotic prescriptions for lower respiratory tract infections in primary care. European Clinical Respiratory Journal. 2018;5(1):1529535.

14. Eom G, Grootendorst P, Duffin J. The case for an essential medicines list for Canada. Canadian Medical Journal. 2016;188(17-18):499-503. 